\title{
L'Oeuvre des livres gratuits dans l'histoire de la lecture publique au Québec (1898-1908)
}

\section{The Influence of L'Oeuvre des livres gratuits (1898-1908) on the Development of Public Reading in Québec L'Oeuvre des livres gratuits (1898-1908) en la historia de la lectura pública de Quebec}

\section{Sophie Montreuil}

Volume 49, numéro 3, juillet-septembre 2003

URI : https://id.erudit.org/iderudit/1030194ar

DOI : https://doi.org/10.7202/1030194ar

Aller au sommaire du numéro

Éditeur(s)

Association pour l'avancement des sciences et des techniques de la documentation (ASTED)

ISSN

0315-2340 (imprimé)

2291-8949 (numérique)

Découvrir la revue

Citer cet article

Montreuil, S. (2003). L'Oeuvre des livres gratuits dans l'histoire de la lecture publique au Québec (1898-1908). Documentation et bibliothèques, 49(3),

129-135. https://doi.org/10.7202/1030194ar
Résumé de l'article

L’Oeuvre des livres gratuits, organisation philanthropique fondée en 1898 par Joséphine Marchand-Dandurand, a expédié des livres sans frais à des particuliers, des institutrices et des bureaux régionaux situés sur l'ensemble du territoire québécois jusqu'à la fin de la décennie 1900. Le projet que caressait sa fondatrice à partir de 1904 était de créer des salles de lecture en province. La gratuité de l'Oeuvre, l'initiative individuelle qui en a été à l'origine, son ouverture à tous ainsi que la moralité des livres qu'elle faisait circuler sont autant de facteurs qui permettent de voir comment l'organisation s'inscrit dans l'histoire de la lecture publique au Québec.
Tous droits réservés (c) Association pour l'avancement des sciences et des techniques de la documentation (ASTED),
Ce document est protégé par la loi sur le droit d'auteur. L’utilisation des services d’Érudit (y compris la reproduction) est assujettie à sa politique d'utilisation que vous pouvez consulter en ligne. 


\title{
L'CEuvre des livres gratuits dans l'histoire de la lecture publique au Québec (1898-1908)
}

\author{
Sophie Montreuil \\ Postdoctorante \\ Projet History of the Book in Canada/Histoire du livre et de l'imprimé au Canada \\ Université McGill \\ sophie.montreuil@mail.mcgill.ca
}

L'CEuvre des livres gratuits, organisation philanthropique fondée en 1898 par Joséphine Marchand-Dandurand, a expédié des livres sans frais à des particuliers, des institutrices et des bureaux régionaux situés sur l'ensemble du territoire québécois jusqu'à la fin de la décennie 1900. Le projet que caressait sa fondatrice à partir de 1904 était de créer des salles de lecture en province. La gratuité de l'CEuvre, l'initiative individuelle qui en a été à l'origine, son ouverture à tous ainsi que la moralité des livres qu'elle faisait circuler sont autant de facteurs qui permettent de voir comment l'organisation s'inscrit dans l'histoire de la lecture publique au Québec.

The Influence of L'CEuvre des livres gratuits (1898-1908) on the Development of Public Reading in Québec

The CEuvre des livres gratuits (1898-1908), a philanthropic organisation founded in 1898 by Joséphine Marchand-Dandurant, shipped books free of charge to individuals, teachers and regional offices all over Québec until 1908. In 1904, the founder wanted to open reading rooms in different areas of the province, offering works, free of charge, that respected the moral code of the day. These factors help appreciate the organisation's role in the development of public reading in Québec.

\begin{abstract}
L'CEuvre des livres gratuits (1898-1908) en la historia de la lectura pública de Quebec

Hasta el fin de la década de 1900, L'CEuvre des livres gratuits, una organización filantrópica fundada en 1898 por Joséphine MarchandDandurand, envió libros gratuitos a particulares, instructores y oficinas regionales situados por lo general en el territorio de Quebec. El proyecto que abrigaba su fundadora a partir de 1904 con la intención de crear salas de lectura en el interior de la provincia, la gratuidad de la obra, el origen de la iniciativa individual, su abertura a todos y la moralidad de los libros que ella hacía circular, son los factores que permiten ver de qué manera esta organización se inscribió en la historia de la lectura pública de Quebec.
\end{abstract}

Q i l'on excepte les villes de Mont-

$\checkmark$ réal et de Québec, dont les diverses sortes de bibliothèques ont été recensées et sont bien documentées ${ }^{1}$, que sait-on de la lecture publique dans le Québec francophone du XIX ${ }^{\mathrm{e}}$ siècle et du tournant du $X^{e}$ siècle? Était-il possible de lire hors des centres urbains, et si oui, comment les livres se rendaient-ils dans les régions éloignées? À quoi les résidents de ces régions pouvaient-ils avoir accès, dans un contexte où le transport des marchandises pouvait être ralenti, voire paralysé durant les longs mois d'hiver? C'est à des questions comme celles-ci que l'histoire de l'CEuvre des livres gratuits, fondée en 1898, par Joséphine Marchand-Dandurand (1861-1925) et en activité pendant au moins une dizaine d'années, permet de donner quelques réponses, suivant l'hypothèse que cette organisation philanthropique s'inscrit dans l'histoire de la lecture publique au Québec, dont elle représente une page peu ou pas connue. Dans cet article, je ne la présenterai que brièvement en mettant l'accent sur la diffusion et la consommation du livre, ainsi que sur l'histoire des bibliothèques au Québec².

\section{Naissance d'une CEuvre}

II n'est pas étonnant de trouver à l'origine de l'CEuvre des livres gratuits le nom de Joséphine Marchand-Dandurand, lectrice passionnée et convaincue de la nécessité de la lecture dans la vie de tous les jours. "Le consolateur, le conseiller toujours prêt, le plus aimable des compagnons dans les moments d'anxiété ou de tristesse, c'est un beau et bon livre. Voilà l'ami parfait dont les bienfaits demeurent, après qu'il est parti ", écrit-elle dans un texte qu'elle reprend et fait paraître en 1901 dans le recueil Nos travers (Dandurand 1901, 24). C'est dans le numéro de janvier 1896 du Coin du feu, le mensuel féminin que J. Marchand-Dandurand lance en 1893 et dont elle tient la barre durant ses quatre années d'existence, que l'on trouve la première trace de l'CEuvre des livres gratuits, qui n'est alors qu'un projet en devenir. Dans sa chronique, elle rapporte avoir reçu une lettre « d'un village perdu à l'extrémité de cette province" où une jeune femme fait état de sa diffi-

1. Voir, entre autres, Lamonde, Yvan. Les bibliothèques de collectivités d̀ Montréal $\left(17^{e}-19^{e}\right.$ siècle) et, 150 ans de lecture publique. 1995. Documentation et bibliothèques 41 (3).

2. Cet article est issu de mes recherches postdoctorales qui sont subventionnées par le projet History of the Book in Canadal Histoire du livre et de l'imprimé au Canada et qui exploitent un fonds d'archives privé et inédit réunissant des sources documentaires variées se rapportant à la vie et à l'Euvre de Joséphine Marchand-Dandurand. Pour l'Euvre des livres gratuits, les sources couvrent les années 1898 à 1908 (cette dernière année ne marque pas nécessairement la fin des activités de l'CEuvre) et comprennent des documents officiels (dont un Rapport produit en 1901 et livré sous forme d'une petite brochure à couverture bleue) et manuscrits de toutes sortes, des coupures de journaux et une correspondance de 238 lettres adressées à l'Euvre par des demandeurs et des donateurs de livres, des inspecteurs d'école, des institutrices et des organisateurs et organisatrices de bureaux régionaux. 


\section{En dehors des bureaux régionaux, les livres sont envoyés à des particuliers, et une offensive visant à rejoindre les institutrices, en passant d'abord par les inspecteurs d'école, est mise en place.}

culté à se procurer des livres: « J'ai un ardent désir de me procurer cet aliment indispensable, mais où trouver celui qui serait en rapport avec mes faibles ressources? Si vous pouviez me renseignez là-dessus, vous auriez donné à mon esprit le verre d'eau dont Dieu certainement vous tiendra compte... ". Cette correspondante anonyme aurait fait naître chez J. Marchand-Dandurand ni plus ni moins qu'une illumination: «la perspective d'une bonne CEuvre à accomplir nous est apparue", et les lectrices du Coin du feu sont appelées en renfortpour créer un réseau de distribution de livres autrement «abandonnés et gaspillés après avoir été lus ${ }^{3}$ ". Le 17 décembre 1897, soit près de deux ans plus tard, J. Marchand-Dandurand fait allusion pour la première fois dans son Journal intime à ce qu'elle nomme sa «bibliothèque des pauvres " (Marchand 2000, 183), qui n'a pas encore officiellement vu le jour, et annonce le 10 janvier 1898 qu'elle entreprend le surlendemain «la distribution des livres aux pauvres" (Marchand 2000, 187). Ce moment marque vraisemblablement le lancement public de l'CEuvre, attesté par une sorte de communiqué, daté du 26 février 1898 , expliquant le bien-fondé de cette sollicitation de livres et précisant où s'adresser pour s'en procurer gratuitement (une adresse qui n'était autre que celle du domicile de J. Marchand-Dandurand). Une campagne de promotion auprès d'éventuels donateurs accompagne la parution de cette annonce dans le journal: dans une lettre datée du $1^{\text {er }}$ mars 1898 , imprimée et reproduite en un nombre indéterminé d'exemplaires, J. Marchand-Dandurand, à titre de secrétaire générale de l'organisation, «se recommande à [la] charité [du destinataire] pour obtenir le don de quelques livres français " et compte sur [son] patriotisme pour aider [l'association] à trouver les provisions » dont elle a besoin.
En novembre et décembre 1898, plusieurs bureaux régionaux sont mis en place à travers le Québec (Chicoutimi, SaintAndré de Kamouraska, Maskinongé, Waterloo, Saint-Hilaire, Saint-Jérôme et Sainte-Cécile du Bic, pour n'en nommer que quelques-uns), et l'CEuvre continue de se développer et de s'étendre géographiquement durant les années suivantes. Elle dépasse rapidement les frontières du Québec, autant pour ce qui est de la provenance des demandeurs de livres (le Rapport de 1901 parle d'envois aux ÉtatsUnis et au Yukon) que des donateurs, qui bénéficient d'une adresse d'expédition à Paris, dès le printemps $1898^{4}$. En dehors des bureaux régionaux, les livres sont envoyés à des particuliers, et une offensive visant à rejoindre les institutrices, en passant d'abord par les inspecteurs d'école, est mise en place, en $1906^{5}$. Un document intitulé Règlement, probablement expédié à qui le demandait ou lors d'un premier envoi, explique les procédures selon lesquelles les livres étaient prêtés: ils pouvaient être conservés pour une durée de quatre mois s'ils étaient dirigés vers un bureau régional, qui recevait en moyenne trois envois par année, et pour une durée de deux mois s'ils étaient adressés à un particulier. Tous les bénéficiaires pouvaient retourner les livres gratuitement en les adressant au ministère des Travaux publics à Ottawa 6 . Le Rapport de 1901 fait état du travail accompli à Montréal par les bénévoles - surtout des femmes - qui accusaient réception des livres et qui en assuraient l'expédition (donateurs et abonnés). Un comité d'ordre et de classement veillait à l'organisation de la bibliothèque ambulante selon un système de classification qui comprenait des « rayons » et des «sections numérotées", lesquels se partageaient les titres d'histoire, de voyage, d'éducation, de religion, les biographies, les romans, etc. ${ }^{7}$ Ce même document révèle l'existence d'un comité d'examen chargé de veiller à la moralité des livres mis en circulation, comité dont la présidence était assumée en 1901 par l'abbé Bourassa, qui succédait au chanoine Dauth.

\section{Une certaine rivalité}

C'est sans doute à l'Aberdeen Association for Distribution of Literature to Settlers in the West, fondée en 1890 par un groupe de femmes de Winnipeg d'après une idée de Lady Ishbel Aberdeen, que J. Marchand-Dandurand emprunte l'idée d'expédier des livres gratuitement à une population établie hors des centres urbains $^{8}$. Lorsqu'elle lance le projet dans Le coin du feu, elle connaît personnellement Lady Aberdeen depuis au moins deux ans et, par l'entremise de sa participation au Conseil national des femmes du Canada, elle est au fait des activités menées par ses consœurs du Canada anglais ${ }^{9}$. En 1898, l'Association Aberdeen compte 14 succursales ou bureaux de distribution qui desservent leur clientèle depuis les villes de Winnipeg, Halifax, Toronto, Ottawa, Calgary, Vancouver, Regina et Montréal, entre autres localisations, cette dernière ville étant la seule à avoir bénéficié d'une double organisation, partagée entre une branche francophone et une branche anglophone. Selon Stewart Mein, l'Association Aberdeen est, au tournant du $X X^{e}$ siècle, « the largest and most

3. Le Coin du feu. janvier 1896.4 (1): 1-2.

4. Parmi les donateurs français, on trouve les noms de Louis Herbette, Jules Siegfried, Gabriel Hanotaux, René Doumic, Hector Fabre et Jules Jusserand.

5. Fondée pour desservir les "habitants des campagnes", l'Euvre comptera ultérieurement dans sa clientèle des gens de la ville, peut-être parce que des demandes de livres provenant de Montréal auront fait comprendre à la secrétaire de l'Euvre que, s'il est en principe plus facile de se procurer des livres en ville, il faut en revanche avoir les moyens de le faire, ce que tous n'ont pas.

6. L'Euvre bénéficiait de la protection de Joseph-Israël Tarte, ministre des Travaux publics sous Laurier (Marchand 2000, 183).

7. Les sources ne comprennent pas de catalogue des titres en circulation, mais il sera possible de reconstruire une liste partielle à partir des annotations manuscrites qui figurent parfois sur les lettres de demandeurs quant aux livres qui leur ont été envoyés, des indications que donnent certains des demandeurs sur les titres reçus, de même que des titres qui ont été offerts à l'Euvre par les donateurs.

8. S'inspire-t-elle de celui donné par les Sulpiciens à la bibliothèque qu'ils mettent sur pied en 1844 , l'Euvre des bons livres, lorsque vient le temps de donner un nom à son association? Elle n'ignorait certainement pas l'existence de cette première "œuvre» liée aux livres.

9. Selon ce qu'elle rapporte de l'événement dans son Journal intime, c'est Lady Aberdeen elle-même qui l'aurait convaincue de prononcer une conférence au congrès annuel de $1894 \mathrm{du}$ Conseil national des femmes du Canada (Marchand 2000, 166). 
important free circulating library in Canada» (Mein 1985, 2).

D'emblée, il faut préciser que l'CEuvre des livres gratuits n'est pas la composante francophone de l'Association Aberdeen, dont la principale responsable est une dame L.D. Provencher, et que, si l'idée ne vient pas d'elle, J. Marchand-Dandurand a par contre présidé seule à la mise en place et au développement de son réseau de distribution. En fait, lorsqu'elle commence à faire la promotion de l'CEuvre, son objectif est d'empêcher qu'on ne confonde les deux associations puisqu'on ne trouve aucune référence à l'Association Aberdeen dans la documentation à caractère officiel sur l'CEuvre des livres gratuits et que, l'article fondateur du Coin du feu, veut faire croire à une création spontanée.

Quelques sources privées montrent, en revanche, que le travail mené en coulisses par J. Marchand-Dandurand a été d'un tout autre ordre, comme en fait foi cette lettre de 1901 de la main de James Sutherland, signée à titre d'Acting Postmaster General, d'après laquelle on comprend que la secrétaire générale de l'CEuvre s'est réclamée ouvertement, au moins une fois mais probablement davantage, de privilèges accordés àl'Association Aberdeen:

"Upon inquiry regarding the Aberdeen Association to which you refer, the only franking privilege they have is that they collect a number of books which are franked by the Department from here for distribution among the settlers in the West, and even this privilege, I fear from the reports, is being abused, and may have to be cancelled ${ }^{10}$."

Une lettre de mars 1898, signée par Auguste Bodard, secrétaire de la Société d'immigration française au Canada, atteste que J. Marchand-Dandurand n'a pas eu d'autre choix que de marcher dans les traces de $\mathrm{M}^{\mathrm{me}}$ Provencher au moment de solliciter des donateurs potentiels ${ }^{11}$.

Selon Stewart Mein, la section montréalaise francophone de l'Association Aberdeen n'expédiait pas de livres dans la province de Québec, mais plutôt dans de petits villages abritant une population d'expression française situés au Manitoba et dans les Territoires du Nord-Ouest (Mein 1985, 12), ce qui nous pousse à

\section{S'il est une chose que l'Euvre des livres gratuits prouve noir sur blanc, c'est un désir de lire à la grandeur du territoire québécois.}

croire que l'CEuvre des livres gratuits n'avait pas de rivale. En principe complémentaires puisque destinées à desservir des marchés distincts, il y a fort à parier qu'une forme de concurrence a néanmoins existé entre les deux associations, d'abord quant à l'approvisionnement en livres français, ce dont témoigne cet extrait d'une lettre d'Alfred Duclos DeCelles, bibliothécaire au Parlement d'Ottawa et membre de l'Association Aberdeen:

"On m'a bombardé l'autre jour viceprésident de l'Association Aberdeen. Mes électeurs ont fait une tête lorsque je leur ai confié que je vous envoyais autant de paquets qu'à la branche française de Montréal! Ils ont fini par comprendre, après explications, que les livres distribués à l'Est faisaient autant de bien que les bouquins répandus dans l'Ouest ${ }^{12}$.»

L'inquiétude qu'a sentie DeCelles de la part des bienfaiteurs de l'Association Aberdeen, qui ne cherchaient sans doute qu'à protéger les sources d'approvisionnement à la base de leur exercice philanthropique, fait écho à celle que manifeste $M^{\text {me }}$ Provencher dans une lettre adressée à J. Marchand-Dandurand durant les premières années d'existence de l'CEuvre des livres gratuits. Cette lettre, qui vise à mettre fin à une situation où les deux associations ont été confondues, montre la possible rivalité entre les deux organisations, qui n'œuvrent pas sur des territoires géographiques aussi éloignés que ne le laisse entendre Mein. «[S] j j'ai contribué à vous mettre sous l'impression que la province de Québec était exclue de notre distribution, je le regrette, car le sous-titre de notre Association dit "for distributing literature to settlers in isolated parts of Canada"13 ». Comme celle à qui elle répond, $M^{m e}$ Provencher veut établir un climat de paix, d'abord en approuvant le geste de J. Marchand-Dandurand de « faire cesser immédiatement ce qui aurait pu créer un malaise entre [elles] », ensuite en souhaitant non pas que les deux organisations s'entraident, mais bien qu'elles délimitent en quelque sorte leur champ d'action: «J'espère comme vous, chère Madame, que nous allons faire le bien sans nous battre jamais, et je suis sûre que nos œuvres peuvent marcher sans se nuire l'une à l'autre, autrement ce serait faire la charité sans avoir de charité, ce qui serait déplorable.» Aucune autre source ne permet d'explorer plus en détail les liens qu'ont pu entretenir les deux organisations, et cette rivalité polie ne demeure pour le moment qu'une hypothèse de travail.

\section{Témoignages d'un contexte}

Dans son essai d'histoire socio-culturelle sur les bibliothèques publiques à Montréal, Marcel Lajeunesse rapporte ces paroles prononcées en 1915 par le Premier ministre Lomer Gouin: «Parce qu'il y a peu de bibliothèques publiques dans la province de Québec, il ne faut pas conclure et il ne faut pas laisser dire que sa population ne lit point ou ne lisait point. ” (Lajeunesse 1996, 190) S'il est une chose que l'CEuvre des livres gratuits prouve noir sur blanc, c'est un désir de lire à la grandeur du territoire québécois, c'est le plaisir de la lecture pratiquée presque au quotidien - en même temps que le manque de livres pour satisfaire ce plaisir. De Saint-

10. Papier en-tête Postmaster General of Canada, 6 juin 1910, adressée au Sénateur Raoul Dandurand. Mein explique que c'est en 1894 qu'est accordé à l'Association Aberdeen, par Sir Adolphe Caron, le "free postage for parcels sent out by the association, up to five pounds in weight » (Mein 1985,5).

11. Les deux femmes ont frappé à la porte des bureaux de l'Alliance française à Paris, $M^{\text {me }}$ Provencher en 1897 et J. Marchand Dandurand en 1898. Papier en-tête Puissance du Canada, 24 mars 1898, lettre adressée au Sénateur Raoul Dandurand.

12. Papier en-tête Library of Parliament, 27 octobre 1898. J. Marchand-Dandurand connaît bien Alfred Duclos DeCelles, qui comble une bonne partie de sa soif de lecture en remettant à Raoul des livres qu'il emprunte pour elle à la Bibliothèque du Parlement.

13. Lettre datée du 23 mars, sans année. 
Sauveur, la jeune Julie Florent écrit à J. Marchand-Dandurand:

"J'ai quatorze ans, mon jeune frère qui est âgé de onze ans doit communier cette année et il demanderait un petit livre $d u$ bon Jésus. Ma petite sour qui a sept ans, vous demande un petit livre d'images. Moi j'aime la science, mais vous nous donnerez ce que vous voudrez. [...] ma famille serait heureuse de lire vos livres, il y en a si peu ici. Le dimanche on lierait cela, ce serait désennuyant.» (2 avril 1898)

Plus âgée, Eulalie Charlebois, depuis la ville de Hull, s'adresse à l'CEuvre en donnant une couleur particulière à sa demande: presque tous célibataires! [...] Le plus grand nombre d'entre nous sait lire et la lecture est à peu près la seule distraction qu'il nous soit donnée ici pendant les soirées et le dimanche. [...] J'ajouterai que, quelques-uns d'entre nous lisons l'anglais. » (25 septembre 1899)

Si ces trois témoignages - auxquels plusieurs autres auraient pu s'ajouter - confirment l'utilité et le caractère divertissant de la lecture auprès d'un échantillon varié de lecteurs, il faut se garder de conclure qu'ils parlent pour l'ensemble de la population québécoise, d'autant plus que quelques-unes des personnes pressenties par J. Marchand-Dandurand pour organiser un bureau régional lui reviennent, à ce sujet, déçues du peu d'intérêt

\section{Dans certains cas, c'est l'existence d'une bibliothèque paroissiale qui explique le manque d'enthousiasme à l'égard de la distribution de livres gratuits.}

"Je viens vous demander aujourd'hui de me permettre de prendre une petite part de ce que vous offrez avec si bonne grâce à tous, mais surtout aux femmes désireuses de perfectionner et de compléter leur instruction, mais dont les modiques revenus sont un empêchement pour un bon nombre d'elles. [...] La bonté avec laquelle, dit-on, vous accueillez ceux qui s'adressent à vous dans ce but, me font [sic] ajouter une autre demande à la première; ce serait de me guider dans le choix d'auteurs qui non seulement récréent mais cultivent l'intelligence et le style de leurs lecteurs et surtout savent nous rendre un peu meilleurs.» (7 juin 1899)

Et que dire de ce dénommé $\mathrm{F}$. Savard, bûcheron de métier « de retour dans les chantiers", qui fait le portrait, depuis River Desert, d'une classe de lecteurs dont on ne soupçonnait peut-être pas l'existence:

"Voici les quelques renseignements que je puis vous donner sur la classe d'hommes que nous sommes: Au nombre de trente-six, canadiens-français, bûcherons, etc. [...] plus ou moins ignorants; nous devons passer l'hiver dans les bois, c['est]-à-d[ire] que nous ne reverrons pas la civilisation avant neuf ou dix mois. Âge: de 17 à 35 ans, soulevé par le projet. «[N]otre population en général est indifférente pour ce qui concerne l'éducation", constate E.L.G. Gauthier depuis Upton (30 octobre 1898); " Il me fait peine de vous dire que je n'ai pas trouvé autant d'enthousiasme que je croyais", se désole Angéline Marchildon de Batiscan (1 $1^{\text {er }}$ avril 1899); "Voilà déjà quelques mois que je ne reçois aucune demande pour des livres après avoir fait annoncer l'CEuvre en chaire, écrit M.A. Lefebvre depuis Waterloo. [...] Je commence à croire que par ici on ne s'occupe pas beaucoup de lire. » (28 décembre 1898)

Dans certains cas, c'est l'existence d'une bibliothèque paroissiale qui explique le manque d'enthousiasme à l'égard de la distribution de livres gratuits ${ }^{14}$ : «J'espérais pouvoir établir ici un bureau [...], mais je n'ai pas pu réussir, écrit L.A. Bertrand de l'Isle-Verte, nous avons une bibliothèque paroissiale qui semble rencontrer tous les besoins de la localité" (4 mai 1899). Le constat est le même à Saint-Joseph d'Alma: «Maintenant je vais être obligée de discontinuer comme ceci, écrit Rosalba Gagné. J'en ai parlé à monsieur le curé dernièrement et comme monseigneur les obligent d'avoir une bibliothèque paroissiale [,] les gens préfèrent s'abonner à cette bibliothèque " (11 octobre 1899) et à Rimouski, ville qui semble particulièrement choyée quant à l'accès aux livres:

"Pour dire le vrai, je crains que vous ne puissiez frapper un grand coup dans notre région, conclut Aimée P. Fiset. Ici, dans la ville, les institutrices sont des religieuses pourvues de livres. Il y a aussi la bibliothèque paroissiale qui contient des beaux livres, choisis par notre ancien curé, le Rnd M.L. Rouleau, un homme intelligent. Ces livres sont à la disposition du public, et sont distribués chaque dimanche à tous ceux qui veulent en profiter. " (14 décembre 1898)

D'après les lettres qui font allusion à la réaction des membres du clergé local devant l'implantation d'un bureau de l'CEuvre des livres gratuits dans leur municipalité, le Révérend Rouleau fait figure d'exception:

"Notre bon Curé Rouleau a laissé Rimouski, poursuit Aimée P. Fiset dans la même lettre, et je vous assure qu'il serait difficile de s'entendre avec le curé actuel, et les bons apôtres qui l'entourent. Ils ont des préjugés contre tout ce qui s'appelle: libéral. Il suffirait que ces livres fussent distribués par des dames dont les maris appartiennent à l'école de Laurier pour qu'ils y voient une répétition de l'histoire des écoles sans Dieu. $»^{15}$.

Près de 20 ans après la fermeture de la bibliothèque de l'Institut canadien de Montréal, le débat qui oppose l'Église catholique et les libéraux au sujet de la lecture publique fait encore rage en province. À Saint-Hyacinthe, où «la meilleure volonté du monde" a accueilli ses premières démarches pour créer un bureau de distribution et où le maire avait accepté de mettre à sa disposition « une très jolie salle à l'Hôtel de ville ", E.F. Beauregard est forcée de tout laisser en plan lorsqu'elle se bute à la fin de non-recevoir que lui signifient les curés de la place:

"À l'Évêché, ces messieurs disent que la chose, n'étant pas sous contrôle religieux [,]

14. Sur les 230 bibliothèques paroissiales qui existent au Québec en 1926, 70 ont été fondées avant 1900 (Lajeunesse 1998,55).

15. L'histoire des "écoles sans Dieu " renvoie à la tentative (avortée) du Premier ministre Félix-Gabriel Marchand de rétablir, dès son arrivée au pouvoir en 1897, le ministère de l'Instruction publique dans ses fonctions, ce qui fit naître une polémique avec $\mathrm{M}^{\mathrm{gr}}$ Bruchési. Raoul Dandurand, qui supportait la réforme depuis Ottawa à titre de sénateur libéral et membre du parti de Wilfrid Laurier, rappelle les événements dans ses $M e ́-$ moires (Dandurand 2000, 86-106). 
serait peut-être tolérée; chez les dominicains, le Révérend Père Roudot, curé, ne s'est pas caché de dire qu'il est absolument opposé à l'Guvre, en principe. "Cette ouvre, dit-il, m'est bien connue, elle a originé en France où elle a été le berceau du socialisme, etc. S'il me tombait sous la main des livres qui ne seraient pas tout à fait à mon goût, je vous dénoncerais certainement $d u$ haut de la chaire.» (21 novembre 1898)

E.F. Beauregard ne poursuivra pas ses démarches pour implanter un bureau dans sa ville, mais, « au risque de prendre rang parmi les socialistes et les révolutionnaires", elle offrira à J. MarchandDandurand de soutenir l'CEuvre d'une autre manière, soit en continuant sa quête de livres, qui «promettait d'être fructueuse». C'est la secrétaire générale de l'organisation qui prendra elle-même les choses en main, une mission qu'elle ne se fera certainement pas prier de remplir, car elle a un argument de taille pour faire céder le clergé local, et surtout le Révérend Père Roudot, avec qui elle a entretenu une brève correspondance. Le 2 décembre 1898, celui-ci lui écrit:

"Avant de répondre à la lettre que m'avez fait l'honneur de m'adresser, j'ai consulté Monseigneur de St-Hyacinthe. Sa grandeur a besoin de réfléchir pour me donner une réponse; personnellement, $\mathrm{Ma}$ dame, j'ai exprimé mon avis sur cette question; l'Euvre a d'excellents côtés, elle peut en voir de très dangereux si un contrôle compétent au point de vue moral et religieux lui manque.»

II est impossible de savoir si cette réponse a été expédiée ou non au Révérend Père Roudot (il s'agit peut-être simplement d'un brouillon), mais une lettre personnelle signée de la main de J. Marchand-Dandurand et datée du 3 décembre 1898 montre qu'elle a néanmoins souhaité devancer le verdict de $\mathrm{M}^{\mathrm{gr}}$ LouisZéphirin Moreau, évêque de Saint-Hyacinthe, et lui couper l'herbe sous les pieds. «La conduite de Mrr Bruchési [dont le Rapport de 1901 indique qu'il compte au nombre des «patrons» de l'CEuvre], écritelle, que vous avez peut-être oublié de signaler à son confrère, aurait pu le dispenser de l'inquiétude de prendre un parti, attendu que ce qui est bon dans un diocèse doit être tolérable dans le voisin." Après avoir tiré la situation au clair, J. Mar-

\section{Gérer une distribution de livres à la grandeur du Québec ne semble pas avoir été chose facile [...] la logistique au jour le jour tenait presque de l'exploit}

chand-Dandurand ne peut s'empêcher de faire la leçon à son correspondant:

"C’est par de pareils procédés que vous faites dire aux ennemis de la religion que vous êtes opposés au développement intellectuel du peuple, écrit-elle. Je ne suis pas de ceux-là [...] et je continuerai avec mes compagnes à faire du bien, là où on nous le permettra, en éclairant les intelligences. Je crois avec $M^{g r}$ Dupanloup [évêque français] qu'on sert mieux le Bon Dieu avec un esprit éclairé que quand on est ignorant et que notre Sainte Religion peut soutenir la lumière.» (3 décembre 1898)

J. Marchand-Dandurand a-t-elle osé mettre à la poste une telle condamnation du comportement d'un membre du clergé? L'appui de $\mathrm{M}^{\mathrm{g}}$ Bruchési nous pousse à penser que oui, mais, parce que la correspondance ne contient aucune autre lettre en provenance de Saint-Hyacinthe, il faut aussi envisager l'hypothèse que le clergé local soit resté sur sa position et qu'il ait ainsi privé la municipalité d'un dépôt de livres gratuits.

Gérer une distribution de livres à la grandeur du Québec ne semble pas avoir été chose facile; si le transport des échanges entre les différents bureaux régionaux pouvaient être ralentis sinon arrêtés temporairement à cause des difficultés saisonnières ou de circonstances imprévues, comme l'apparition de «Dame Picote» qui paralyse l'organisation pendant quelques semaines à l'hiver $1902^{16}$, la logistique au jour le jour tenait presque de l'exploit, comme en fait foi ce compte rendu adressé à J. Marchand-Dandurand par E.B. Mercier, l'une de ses bénévoles montréalaises:

"Je ne comprends pas comment cela se fait que $M^{\text {lle }} R$. Gagné ait reçu des livres. Vous lui avez donnée [sic] ordre d'envoyer les siens à $M^{\text {lle }} C$. Rioux, et j'ai écrit à $M^{\text {lle }} L$. Delisle d'envoyer ses livres à Chicoutimi à $M^{m e}$ Savard. J'écris à St-Jérôme demandant à $M^{l l e}$ Delisle si elle à [sic] reçu un nouvel envoi de livres; et à qui elle a envoyé le premier envoie [sic]. S'il y avait erreur vous pourriez peut-être donner ordre à $M^{\text {lle }}$ Desjardins d'envoyer à $M^{\text {me }}$ Savard, le dernier envoi de livres qu'elle à [sic] reçu.» (7 novembre 1899)

Un tel bilan permet de prendre la juste mesure du travail accompli pour mener à terme l'envoi de chacun des 700 paquets, « représentant de sept à huit milles [sic] volumes ou revues", dont fait état le Rapport de 1901. Les défis que J. Marchand-Dandurand et ses consœurs durent relever n'étaient cependant pas de nature à les décourager, puisque, durant la seconde moitié des dix années d'existence de l'CEuvre, l'organisatrice en chef tente de donner un nouveau souffle à son association. Une lettre imprimée, datée du 18 juillet 1904, annonce son désir de fonder de véritables «salles de lecture» et de « constituer un stock roulant, pour le distribuer entre un certain nombre de bureaux qui en feraient l'échange entre eux ». On peut s'interroger sur la nature de l'«évolution» annoncée ici, qui ne semble pas différer du fonctionnement régulier de l'organisation. J. Marchand-Dandurand croit cependant le contraire, car elle explique que « ce projet implique une sélection de matières à lire plus systématique que celle qui nous a servi jusqu'ici à satisfaire notre clientèle éparse "; suit une énumération des types d'ouvrages ou d'«éléments qui conviendraient aux commencements de bibliothèques" qu'elle veut établir. Peut-être reprend-elle ici l'idée que tentait de lui faire adopter un petit consortium de donateurs français au tout début de la mise sur pied de l'CEuvre? Dans sa lettre du 24 mars 1898, Auguste Bodard, en faisant référence à une demande de livres adressée à Juliette Lambert Adam ${ }^{17}$,

16. L'information aurait été annoncée dans La Patrie (Laurentides, lettre de Samuel Goulet, 26 janvier 1902).

17. Femme de lettres française (1836-1936), militante féministe, fondatrice de La Nouvelle Revue (Paris, 1879-1940) elle tenait un salon fréquenté par de nombreux écrivains et hommes politiques sous la III République. 


\section{l'organisation n'est pas le fruit d'une initiative collective, celle du clergé, des artisans, des ouvriers ou des gens de profession libérale, mais bien d'une initiative individuelle}

portait ainsi cette suggestion à l'attention de J. Marchand-Dandurand:

"[A] $\mathrm{u}$ lieu de donner des livres aux particuliers sans profit pour l'intérêt public, ils [tous les amis et connaissances auxquels elle en avait déjà parlé, notamment M. Louis Herbette, conseiller d'État] aimeraient mieux [...] que l'Alliance française à laquelle Madame Provencher en a déjà demandé l'année dernière, à ma suggestion, aide Madame Dandurand à fonder des bibliothèques publiques dans les paroisses ou colonies de langue française de l'Ouest du Canada.»

Cette hésitation n'empêchera pas plusieurs donateurs français de faire parvenir des livres à l'CEuvre des livres gratuits en leur nom personnel, dont Juliette Adam elle-même, qui se permettra toutefois de formuler au moins une fois à J. Marchand-Dandurand le commentaire suivant, en post-scriptum: «Si vous fussiez une bibliothèque on pourrait provoquer des dons imposants parce que les donateurs se diraient que les ouvrages ne sont pas éparpillés. " (Paris, 30 mai 1898)

Ce projet de salles de lecture régionales refait surface en avril 1906, dans une lettre de la main de J. Marchand-Dandurand, encore une fois soit à l'état de brouillon ou à titre de missive n'ayant jamais été expédiée. II est cependant quelque peu modifié, puisqu'il se rattache cette fois à l'offensive menée en février de cette même année en direction des institutrices qui exercent leur métier en région. Dans un exposé à caractère très officiel, adressé à un anonyme «Dear Sir» - la lettre est rédigée en anglais -, J. Marchand-Dandurand sollicite la bienveillance de son correspondant à l'égard de l'entreprise suivante: "Ourprovince is divided into 35 or forty school districts, having each an inspector. My idea would be to found in each district, a regular circulating library, under the supervision of the inspector. 》 (28 avril 1906) Elle ne donne pas de détails sur le fonctionnement de cette "circulating library»-les abonnés auraient-il dû débourser des frais pour en être membres? -, mais il est probable que ce à quoi elle pense tienne davantage de la bibliothèque ambulante gratuite, dans l'esprit de son CEuvre, que de la bibliothèque de location, et ce, d'autant plus qu'elle met l'accent sur la nature essentiellement philanthropique de ses réalisations: "Depending upon charity and privileges, écrit-elle, I have, in past years, managed to distribute a certain amount of books sometimes reaching five or 6000 a year. " Parce qu'elle pense à un réseau mettant à profit les «school districts", il faut aussi se demander si J. MarchandDandurand ne s'inspire pas ici du modèle des bibliothèques scolaires ontariennes, qui, depuis la loi scolaire de 1850, (initiative du révérend Egerton Ryerson à titre de surintendant de l'instruction publique au Haut-Canada) s'ouvrent aux élèves mais aussi à l'ensemble de la population des municipalités et sont financées dans une proportion égale par l'État et par les citoyens.

\section{Les premiers pas d'une marche vers la bibliothèque publique}

Ce projet, sous cette forme ou tel que proposé en 1904, a-t-il vu le jour? Vraisemblablement pas. D'une part parce qu'aucune autre source documentaire n'y fait référence, d'autre part parce qu'il aurait certainement laissé des traces sur lesquelles des chercheurs seraient tombés et que l'histoire nous en aurait révélé l'existence depuis. Cela dit, bien qu'elle n'ait jamais eu la forme d'une «vraie» bibliothèque il ne fait aucun doute que l'CEuvre des livres gratuits fasse partie intégrante de l'histoire de la lecture publique au Québec.

Certes marginale, par rapport aux bibliothèques de collectivités qu'Yvan Lamonde place à l'origine de la «marche vers la bibliothèque publique " (Lamonde 2004), l'organisation de J. MarchandDandurand n'en demeure pas moins intimement liée au mouvement qui connaîtra son premier aboutissement avec la fondation de la Bibliothèque municipale de Montréal, en 1917.

Avant d'explorer cette question plus attentivement en conclusion, voyons d'abord les quatre caractéristiques qui me semblent résumer la nature singulière de l'Cuuvre des livres gratuits, à la fois originale et de son temps.

La gratuité des livres est sans contredit l'aspect sur lequel il faut d'abord insister, ne serait-ce que pour respecter la volonté de J. Marchand-Dandurand de l'inscrire d'emblée dans le nom de l'CEuvre. Contrairement à ce qui fait apparaître et exister les bibliothèques de collectivités, cette gratuité est essentiellement le fruit d'une activité philanthropique, où les riches sont appelés à donner aux pauvres. Ainsi, seuls les dons des bienfaiteurs sont ainsi en mesure d'assurer la survie de l'CEuvre, une situation à laquelle J. Marchand-Dandurand tentera de remédier à plusieurs reprises en essayant de trouver des sources de financement publiques, telles la Ville de Montréal et le gouvernement provincial ${ }^{18}$.

En deuxième lieu, rappelons que, si elle vise d'abord les habitants des régions - même si elle s'ouvre assez rapidement aux résidents de Montréal -, l'CEuvre des livres gratuits ne pratique, par ailleurs, aucune autre forme de discrimination: tous, jeunes et plus âgés, hommes et femmes, célibataires ou mariés, peuvent demander des livres et en obtenir. Elle a été fondée expressément pour alimenter tous ceux qui n'ont pas accès au livre par l'entremise des bibliothèques et non pour satisfaire les besoins des membres d'une association ou d'une corporation professionnelle.

Dans le même ordre d'idées, l'organisation n'est pas le fruit d'une initiative collective, celle du clergé, des artisans, des ouvriers ou des gens de profession libérale, mais bien d'une initiative individuelle; celle d'une femme qui décide de doter sa province d'un merveilleux outil d'instruction et de divertissement, voire de résistance à la menace anglophone.

18. Brouillon d'une lettre sans date adressée au Conseil de ville, où J. Marchand-Dandurand demande une "modeste allocation», et lettre du Premier ministre Lomer Gouin, où celui-ci fait allusion à une demande de subvention en rapport avec l'Euvre des livres gratuits (papier en-tête Cabinet du Premier ministre, 2 janvier 1907). 
Par son caractère ambulant, la bibliothèque qu'elle met sur pied demande en revanche la collaboration des bénéficiaires, qui participent activement au bon déroulement des opérations et qui sont au moins aussi utiles à l'CEuvre que ne le sont les bienfaiteurs.

Enfin, l'CEuvre des livres gratuits ne distribue à ses protégés que des livres d'une parfaite moralité, ce qu'atteste, entre autres, le comité d'examen présidé par un membre du clergé ${ }^{19}$. La lecture, dont l'CEuvre fait la promotion, - et non seulement celle des femmes, comme l'expérience du Coin du feu pourrait donner à le penser - est une activité qui doit être encadrée, supervisée par une autorité capable d'éviter que des livres «immoraux » ne soient mis en circulation. À cet égard, l'CEuvre des livres gratuits s'insère pleinement dans le concept de la lecture partagée. La lecture en elle-même n'est pas condamnable pour grand nombre de personnes au Québec au début du XX $X^{\ominus}$ siècle (comme c'était le cas au XIXe siècle), mais ce sont les «mauvaises » lectures qui sont condamnées. (Savard 1997, 22).

Le retard qu'accuse le Québec par rapport au reste du continent nord-américain quant au «Public Library Movement » n'est aujourd'hui plus à prouver. Durant la seconde moitié du $\mathrm{XIX}^{\mathrm{e}}$ siècle, et dans le sillage du phénomène qui se manifeste aux États-Unis dès le milieu du siècle, le Canada anglais se dote d'un réseau de lecture publique qui commence à peineà apparaître au Canada français au tournant du XXe siècle. En 1882, la loi ontarienne sur les bibliothèques gratuites (Free Libraries Act) donne aux municipalités le plein pouvoir de décision en matière de bibliothèques, mais leur impose en échange d'assurer entièrement la responsabilité de leur financement et des les ouvrir gratuitement au public. La loi est révisée en 1895 pour devenir une loi sur les bibliothèques publiques (Public Library Act). Celle-ci facilitera l'établissement de bibliothèques municipales sur le plan administratif. Dans le même esprit, le Québec adopte en 1890 - un an avant la Colombie britannique - , sous le gouvernement libéral d'Honoré Mercier, une loi qui autorise les municipalités à se doter de bibliothèques gratuites, mais cette loi n'aura de répercussions réelles que chez les anglophones, qui fondent une bibliothèque publique à Knowlton en 1894. Suivra celle de Westmount en 1899 et à Waterloo en 1900 , alors que Montréal devra attendre jusqu'en 1917 pour voir s'ouvrir la première bibliothèque municipale francophone gratuite.

C'est l'Église catholique qui, à partir de la décennie 1840, ralentit considérablement la «marche vers la bibliothèque publique" dans le Québec francophone, entre autres, en mettant sur pied son propre réseau de bibliothèques paroissiales, créées pour contrer l'apparition de bibliothèques non placées sous l'égide du clergé (Lajeunesse 1998, 64). Comme elle s'est manifestée à l'égard de la bibliothèque de l'Institut canadien de Montréal, l'opposition de l'Église au projet d'une bibliothèque municipale dans cette même ville est virulente, autant lorsque l'idée est lancée par le maire Raymond Préfontaine en 1901 que lorsque l'échevin Honoré Mercier fils la relance en 1908 (Lajeunesse 1996), donnant alors lieu à un différend public entre $\mathrm{M}^{\mathrm{gr}}$ Bruchési et Raoul Dandurand (Dandurand 2000, 113-118). Cette opposition féroce nous porte à croire que, malgré la protection de $\mathrm{M}^{\mathrm{gr}}$ Bruchési dont elle bénéficiait, l'CEuvre des livres gratuits aurait difficilement pu dépasser son statut d'entreprise philanthropique privée pour devenir un véritable réseau de bibliothèques provinciales autonomes, administré par des laïcs, tant le clergé n'était pas prêt à céder sa place en matière de contrôle de la lecture publique. Dans le même ordre d'idées, la correspondance de l'CEuvre des livres gratuits, qui atteste l'existence de bibliothèques paroissiales dans plusieurs villes québécoises, montre par ailleurs que ces bibliothèques étaient parfois en mesure de satisfaire les besoins des citoyens et qu'elles contribuaient ainsi à limiter le champ d'action de l'organisation.

Pourquoi l'CEuvre des livres gratuits a-t-elle cessé un jour d'exister? Les sources documentaires n'offrent pas de réponse à cette question, qui demeure dès lors ouverte. Peut-être la fondatrice, dont les activités seront ralenties par la maladie durant les quinze dernières années de sa vie, l'a-t-elle délaissée graduellement pour s'occuper à autre chose ou peut-être a-t-elle été simplement découragée à la longue par les subsides publics qui ne venaient pas? Peut-être les donateurs se sont-ils rarifiés? Peut-être les bibliothèques paroissiales, dont 43 sont fondées entre 1900 et 1909 et 56 entre 1910 et 1919 (Lajeunesse 1998, 55), ont-elles mieux rempli leur rôle?

Que J. Marchand-Dandurand n'ait pas été en mesure de développer l'CEuvre des livres gratuits comme elle l'aurait souhaité n'enlève rien au rôle appréciable que l'organisation a joué dans l'émergence de la lecture publique au Québec, surtout dans la vie de tous les jours d'une population dont $\mathrm{M}^{\mathrm{me}}$ Ferdinand Marcotte de Warwick se fait la porte-parole: « Dans les villes où les livres sont si abondants, on ne peut se faire une idée juste de ce qu'on peut souffrir par privation de lecture; tout en n'étant pas assez fortunée pour achetter [sic] des livres, j'ai cependant des loisirs, de longs dimanches, de longues soirées où un volume me serait si agréable. 》 (8 février 1906) ]

\section{Sources consultées}

Dandurand, $\mathrm{M}^{\mathrm{me}}$ [Joséphine Marchand]. 1901. Faut-il lire. In Nos travers. Montréal: Beauchemin, p. 22-25.

Dandurand, Raoul. 2000. Mémoires (1861-1942), édités par Marcel Hamelin. Québec: Presses de l'Université Laval. $352 \mathrm{p}$.

1995. 150 ans de lecture publique. Documentation et bibliothèques 41 (3)

Lajeunesse, Marcel. 1996. Les bibliothèques publiques à Montréal au début du XX $X^{\circledR}$ siècle: essai d'histoire socio-culturelle. In Readings in Canadian Library sous la direction de Peter F. McNally. Ottawa: Canadian Library Association, p. 173-198.

Lajeunesse, Marcel. 1998. Les bibliothèques paroissiales, précurseurs des bibliothèques publiques au Québec? In Les bibliothèques québécoises d'hier à aujourd'hui. s.l.: Asted, p. 43-66.

Lamonde, Yvan. 1979. Les bibliothèques de collectivités à Montréal (170-19॰ siècle). Montréal: Bibliothèque nationale du Québec. $139 \mathrm{p}$.

Lamonde, Yvan. 2004 (à paraître). Canadian Print and the Emergence of a Public Culture in the Eighteenth and Nineteenth Centuries. In Les idées en mouvement: perspectives en histoire intellectuelle et culturelle au Canada, sous la direction de DamienClaude Bélanger, Sophie Coupal et Michel Ducharme. Québec: Presses de l'Université Laval, p. 175-190.

Marchand, Joséphine. 2000. Journal intime 1879-1900, édition préparée et annotée par Edmond Robillard. Montréal: Éditions de la Pleine Lune. 275 p.

Mein, Stewart G. 1985. The Aberdeen Association: An Early Attempt to Provide Library Services to Settlers in Saskatchewan. Saskatchewan History 38 (1) : 2-19.

Savard, Réjean. 1997. Le discours sur la lecture et l'évolution des bibliothèques au Québec de 1850 à 1950. Argus 26 (2): 19-27.

19. Les œuvres d'imagination ne sont pas interdites, mais il doit s'agir de «bons » romans (lettre de J.Marchand-Dandurand du 18 juillet 1904). 